\title{
Surgeons lack predictive accuracy for anastomotic leakage in gastrointestinal surgery
}

\author{
A. Karliczek • N. J. Harlaar • C. J. Zeebregts • \\ T. Wiggers • P. C. Baas • G. M. van Dam
}

Accepted: 20 January 2009 /Published online: 17 February 2009

(C) The Author(s) 2009. This article is published with open access at Springerlink.com

\begin{abstract}
Background The dramatic clinical consequences of anastomotic leakage in gastrointestinal surgery can be reduced by a diverting stoma or drainage of the peri-anastomotic area. Currently, the surgeons' clinical judgement is of major importance in decision making, but reliable data of the diagnostic accuracy are lacking. In this prospective clinical study, the surgeons' predictive accuracy for anastomotic leakage was evaluated.

Materials and methods In 191 patients undergoing colorectal resection with anastomosis, the risk for anastomotic leakage was determined by the surgeon on the basis of a visual analogue scale (VAS). This risk assessment was compared to the actual occurrence of anastomotic leakage post-operatively.

Results A total of 26 (13.6\%) patients showed anastomotic leakage. The surgeons' median predicted leakage rate was $7.1 \%$ in anastomoses $>15 \mathrm{~cm}$ from the anal verge and $9.5 \% \leq 15 \mathrm{~cm}$ (sensitivity $38 / 62 \%$, specificity $46 / 52 \%$ ). Diagnostic accuracy was not influenced by the surgeons' training level (VAS score,
\end{abstract}

A. Karliczek $\cdot$ N. J. Harlaar $\cdot$ C. J. Zeebregts $\cdot$ T. Wiggers •

G. M. van Dam $(\bowtie)$

Department of Surgery, University Medical Center Groningen, P.O. Box 30001, 9700 RB Groningen, The Netherlands

e-mail: g.m.van.dam@chir.umcg.nl

A. Karliczek • P. C. Baas

Department of Surgery, Martini Hospital Groningen,

Groningen, The Netherlands

N. J. Harlaar • G. M. van Dam

BioOptical Imaging Center Groningen (BICG),

University of Groningen,

Groningen, The Netherlands surgeons $7.8 \%$ vs assistant surgeons $8.5 \%, p=0.96$, sensitivity $41 \%$ vs $44 \%$, specificity $59 \%$ vs $48 \%, p=0.20$ ).

Conclusion The surgeons' clinical risk assessment appeared to have a low predictive value for anastomotic leakage in gastrointestinal surgery. The low a priori risk of anastomotic leakage of $14 \%$ resulted in a low post-test odds (11\%) of correct prediction of anastomotic leakage. This warrants the ongoing search for a better diagnostic test of anastomotic leakage to prevent morbidity and mortality.

Keywords Risk assessment - Colorectal anastomosis · Anastomotic leakage $\cdot$ Clinical complication estimation

\section{Introduction}

Gastrointestinal anastomotic leakage causes increased early mortality [1] and, following potentially curative resection of colorectal cancer, leads to higher recurrence rates and a poorer oncologic prognosis [2]. Clinically relevant anastomotic leakage rates range between $3 \%$ and $19 \%$ [1, 3-9]. Anastomotic leakage may remain localized, causing perianastomotic inflammation or abscess formation, or may progress to generalised peritonitis. Several authors have demonstrated that a diverting stoma, placed in the proximal colon or ileum during the initial operation, prevents anastomotic leakage [9] and clearly reduces the incidence of generalised peritonitis and thus reoperations, intensive care unit (ICU) stay and mortality [10, 11]. In pelvic anastomosis, drainage might reduce the consequences of anastomotic leakage [11], similar to oesophageal anastomosis within the thoracic cavity. Given these possibilities to reduce the major consequences of anastomotic leakage during the initial operation, the operating surgeon might thus decide to construct a diverting stoma in case of high 
risk for anastomotic leakage if determined by a reliable predictive test.

So far, little is known about the accuracy of the clinical prediction of the risk of anastomotic leakage by the operating surgeon. Three studies have shown that the surgeons' global assessment is a predictor of complications in general $[12,13]$ and is even more accurate than risk assessment by Physiological and Operative Severity Score for the Enumeration of Mortality and Morbidity (POSSUM) scores [14]. Until now, no studies have been published evaluating the surgeons' clinical judgement on the risk of anastomotic leakage. The aim of the present study is to assess the accuracy of the surgeons" "gut feeling" for the occurrence of anastomotic leakage on the basis of a visual analogue scale.

\section{Materials and methods}

Patients undergoing gastrointestinal resection in a large teaching hospital and a University hospital between August 2006 and August 2007 were studied prospectively. After completing the operation, the surgeon was asked to predict the risk of clinically relevant anastomotic leakage on a visual analogue scale (VAS) as shown in Fig. 1. Data on pre- and intra-operative risk factors for anastomotic leakage and post-operative complications were prospectively collected by review of patient files during hospital stay and after visit at the outpatient clinic (Table 1).

Anastomotic leakage was considered to be present: (1) when described at relaparotomy or endoscopy, (2) when post-operative computerized tomography scan showed the presence of air or fluid collections or an infiltrate surrounding the anastomosis. Follow-up on anastomotic leakage was continued until 3 months after the initial operation or until discharge.

Of 242 patients, a total of 51 patients (21\%) were excluded from further analysis (Fig. 2). In 12 (5\%) patients, no anastomosis was performed; in $37(15 \%)$ no risk prediction VAS was filled out within $24 \mathrm{~h}$ after the operation. These patients did not differ in respect to the number of anastomotic leakages or in general risk factors. Two cases were excluded as their records on post-operative complications were missing. The remaining 191 patients were all included in the analysis.

\section{Risk of clinically relevant anastomotic leakage}

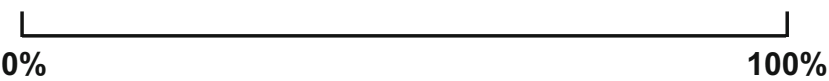

Fig. 1 Visual analogue scale for prediction of risk for anastomotic leakage to be marked by the surgeon immediately after completing the operation
Table 1 Risk factors for anastomotic leakage analysed by univariate analysis

Age

Gender

Diagnosis

Tumour stage

Diabetes

Pulmonary disease

Cardiovascular disease

Smoking

Weight loss

Body mass index

Corticosteroid use

American Society of Anesthesiologists' level

Preoperative irradiation

Weight loss

Peritonitis

Bowel preparation

Intra-operative adverse events

Laparoscopic procedure

Type of anastomosis

Hand-sutured/stapled anastomosis

Distance form anal verge

Mobilization of splenic flexure

Drain near anastomosis

Defunctioning stoma

Duration of operation

Blood loss

Blood transfusion

Intra-operative hypotension

Intra-operative use of vasoactive drugs

Emergency operation

Educational level surgeon

Surgeon

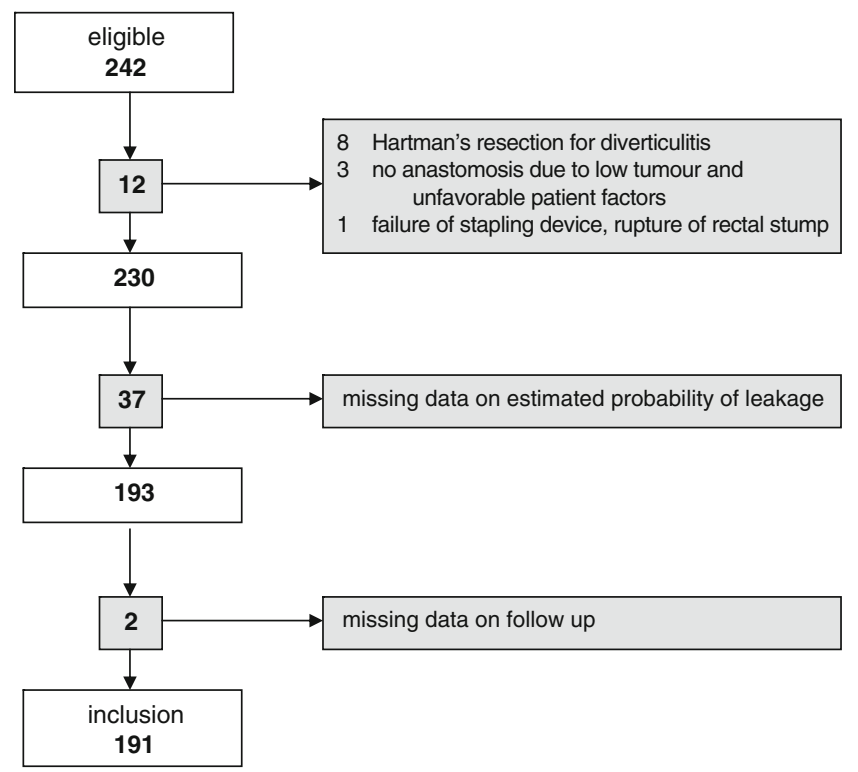

Fig. 2 Flow chart of included patients. Bold numbers indicate patient numbers. Gray boxes indicate excluded patients 
Table 2 Patient characteristics of included patients $(n=191)$

\begin{tabular}{|c|c|c|c|}
\hline & \multicolumn{2}{|c|}{ Anastomotic leakage } & \multirow[t]{2}{*}{$p$} \\
\hline & No $(n=164)$ & Yes $(n=27)$ & \\
\hline Mean (SD) age (years) & $66(14)$ & $62(14)$ & 0.14 \\
\hline \multicolumn{4}{|l|}{ Sex } \\
\hline Male & $91(55)$ & $11(41)$ & 0.16 \\
\hline Female & $73(45)$ & $16(59)$ & \\
\hline Nicotine abuse & $32(20)$ & $7(26)$ & 0.53 \\
\hline Diabetes & $21(13)$ & $1(4)$ & 0.17 \\
\hline Corticosteroid use & $12(73)$ & $3(11)$ & 0.51 \\
\hline Cardiovascular disease & $44(27)$ & $11(41)$ & 0.15 \\
\hline Pulmonary disease & $32(20)$ & $6(22)$ & 0.77 \\
\hline American Society of Anesthesiologists' level & & & 0.27 \\
\hline I & $50(31)$ & $5(19)$ & \\
\hline II & $92(56)$ & $16(59)$ & \\
\hline III & $21(13)$ & $6(22)$ & \\
\hline \multicolumn{4}{|l|}{ IV } \\
\hline Acute operation & $10(16)$ & $2(7)$ & 0.80 \\
\hline Diagnosis & & & 0.22 \\
\hline Malignancy & $110(67)$ & $17(63)$ & \\
\hline Diverticular disease & $23(14)$ & $4(15)$ & \\
\hline Ulcerative colitis/Crohns' disease & $8(5)$ & $3(11)$ & \\
\hline Adenoma & $9(5)$ & $1(4)$ & \\
\hline Continuity restoration (intra-peritoneal) & $9(5)$ & $0(0)$ & \\
\hline Miscellaneous & $5(3)$ & $2(7)$ & \\
\hline Neoadjuvant treatment & & & 0.01 \\
\hline None & $142(87)$ & $16(64)$ & \\
\hline 25 Gy irradiation & $18(11)$ & $7(28)$ & \\
\hline Chemotherapy $+50 \mathrm{~Gy}$ & $3(2)$ & $2(8)$ & \\
\hline \multicolumn{4}{|l|}{ Level of anastomosis } \\
\hline Colon & & & 0.37 \\
\hline Ascending and transverse & $59(36)$ & $6(22)$ & \\
\hline Descending and sigmoid & $42(26)$ & $8(30)$ & \\
\hline Pelvic ( $\leq 15 \mathrm{~cm}$ from anal verge) & $63(38)$ & $13(48)$ & \\
\hline Mean height in $\mathrm{cm}(\mathrm{SD})$ & 13.9 & 10.3 & 0.04 \\
\hline \multicolumn{4}{|l|}{ Main operator } \\
\hline Assistant & $84(51)$ & $9(33)$ & 0.09 \\
\hline Surgeon & $80(49)$ & $18(67)$ & \\
\hline Laparoscopic procedure & $19(12)$ & $1(4)$ & 0.28 \\
\hline Stapled anastomosis & $64(39)$ & $14(52)$ & 0.22 \\
\hline Defunctioning stoma & $11(7)$ & $6(22)$ & 0.01 \\
\hline Mobilization of splenic flexure & $35(21)$ & $11(41)$ & 0.04 \\
\hline Duration of procedure in min (SD) & $174(67)$ & $204(91)$ & 0.06 \\
\hline Blood loss in ml (SD) & $283(284)$ & $464(442)$ & 0.01 \\
\hline Number of packed cells units transfused (SD) & $0.3(0.9)$ & $0.4(0.9)$ & 0.56 \\
\hline
\end{tabular}

The number of patients (\% within leakage-non leakage) is shown

Data were analyzed with Statistical Package for the Social Sciences software (SPSS 15-0, SPSS, Chicago, IL, USA). The data are presented as means (SD) unless indicated otherwise. Differences between categorical variables were tested with Pearson's $\chi^{2}$ test. Differences between continuous variables were tested with Student's two-tailed test (normal distribu- tion) or Mann-Whitney $U$ test (skewed distribution). The influence of pre- and intra-operative patient-related factors on the occurrence of anastomotic leakage was investigated by means of univariate analysis. Variables that had a direct influence on anastomotic leakage after univariate analysis $(p<0.1)$ were entered into a multivariate regression model 
Table 3 Post-operative complications

\begin{tabular}{lc}
\hline Complications & $n=191$ \\
\hline Anastomotic leakage & \\
No & $165(86 \%)$ \\
Yes & $26(14 \%)$ \\
Wound infection or dehiscence & \\
No & $162(85 \%)$ \\
Yes & $24(13 \%)$ \\
Missing & $5(2 \%)$ \\
Cardiac complications & \\
None & $169(89 \%)$ \\
Ischemic & $2(1 \%)$ \\
Arrhythmias & $4(2 \%)$ \\
Decompensation & $11(6 \%)$ \\
Missing & $4(2 \%)$ \\
Pulmonary complications & \\
None & $169(84 \%)$ \\
Pneumonia & $12(6 \%)$ \\
Pleural effusion & $7(4 \%)$ \\
Miscellaneous & $4(2 \%)$ \\
Missing & $8(4 \%)$ \\
\hline
\end{tabular}

The number of patients is shown; between parentheses percentage of all patients

and analysed in a stepwise backward manner. $p$ values $<0.05$ were regarded as statistically significant.

\section{Results}

A total of 191 patients was analysed (patient characteristics are shown in Tables 2 and 3 ). Five $(2.6 \%)$ patients died during the study period. In three of these patients, anastomotic leakage was present; two died of other causes. Twenty-six patients (13.6\%) showed anastomotic leakage. In six cases $(3 \%)$, leakage occurred in ascending and transverse colon anastomoses, eight (4\%) in left colon anastomoses, and $13(7 \%)$ in anastomoses within $15 \mathrm{~cm}$ of the anal verge. In $21(81 \%)$ patients with anastomotic leakage, a re-operation was performed. Duration of ICU stay (0.5 SD 3 days vs 8 SD 12 days, $p \leq 0.05$ ) and hospital admission (11 SD 5 days vs 45 SD 41 days, $p \leq 0.05$ ) were significantly longer after anastomotic leakage.

The operations were performed by a total of 32 different surgeons, either in the role of first operator or as an assistant surgeon or supervisor. If an assistant surgeon was the first surgeon for a procedure (93/48\%), this was always supervised by a staff surgeon. Of all 191 operations, 151 (79\%) were by an assistant supervised by a gastrointestinal surgeon, and in 75 (39\%) operations, a gastrointestinal surgeon was the first surgeon. Compared to assistant surgeons, anastomoses performed by surgeons showed more anastomotic leakage $(10 \%$ vs $18 \%, p=0.09)$. The visual analogue scale recordings showed a median estimated probability for anastomotic leakage of $7.8 \%$ with an uneven distribution (mean 9.5 SD 6.2\%, range 0.7-29.1\%), as shown in Fig. 3. There were no significant differences between surgeons and assistant surgeons in respect to visual analogue scale score $(7.8 \%$ vs $8.5 \%, p=0.96)$. For anastomoses in the ascending and transverse colons, the median estimated probability of leakage was $5.7 \%$ SD $4.7 \%$; in the descending and sigmoid colons $10.3 \% \mathrm{SD}$ $6.0 \%$; and in the rectum ( $\leq 15 \mathrm{~cm}$ from the anal verge) $9.5 \%$ SD $6.7 \%$.

Patients showed more often anastomotic leakage when a defunctioning stoma was constructed $(6 / 35 \%)$, compared to patients without stoma $(21 / 12 \%, p=0.01)$. The estimated risk by the surgeon for anastomotic leakage was $11.80 \%$ (SD 7.9\%) when a stoma was constructed vs 9.3\% (SD $6.0 \%)$ without a stoma $(p=0.28)$. In patients with an anastomosis within $15 \mathrm{~cm}$ of the anal verge $(n=76)$, seven (26.9\%) patients showed more often leakage after mobilisation of the splenic flexure, compared to six $(12.2 \%)$ patients without mobilisation of the splenic flexure $(p=$ 0.01). No significant difference in estimated risk for anastomotic leakage was seen when comparing mobilisation of the splenic flexure vs no mobilisation $(11.8 \% \mathrm{SD}$ $7.5 \%$ vs $10.6 \%$ SD $6.3 \%, p=0.47$ ).

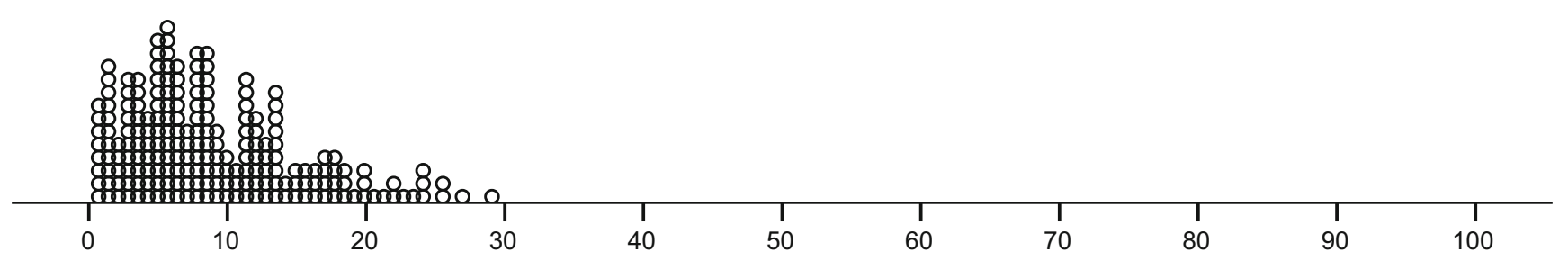

Fig. 3 Histogram representing estimated risk of anastomotic leakage (VAS score) as recorded by the operating surgeon. The cumulative number is shown on the $y$-axis, the $x$-axis shows the estimated percent of leakage 
Table 4 Factors associated with anastomotic leakage at univariate analysis $(n=191)$ and entered into multivariate analysis

\begin{tabular}{|c|c|c|c|}
\hline & No leakage & Leakage & $p$ \\
\hline Anastomosis $>15 \mathrm{~cm}$ & $n=102$ & $n=13$ & \\
\hline Tumour stage & $2.4(0.9)$ & $3.0(0.6)$ & $0.05^{\mathrm{a}}$ \\
\hline Weight loss & $20(20 \%)$ & $5(38 \%)$ & $0.09^{\mathrm{b}}$ \\
\hline Body mass index (SD) & $26.4(4.6)$ & $24.3(4.7)$ & $0.13^{\mathrm{a}}$ \\
\hline Total pre- and intra-operative risk factors (SD) & $3.8(2.0)$ & $4.9(2.7)$ & $0.08^{\mathrm{a}}$ \\
\hline Duration of operation (min) & $148(53)$ & $213(115)$ & $0.08^{\mathrm{a}}$ \\
\hline Blood loss (ml) (SD) & $212(251)$ & $363(342)$ & $0.08^{\mathrm{a}}$ \\
\hline American Society of Anesthesiologists' classification (SD) & $1.9(0.7)$ & $2.2(0.6)$ & $0.07^{\mathrm{a}}$ \\
\hline Anastomosis $<15 \mathrm{~cm}$ & $n=63$ & $n=13$ & \\
\hline Cardiovascular disease & $13(20 \%)$ & $6(46 \%)$ & $0.06^{\mathrm{b}}$ \\
\hline Preoperative irradiation & $20(32 \%)$ & $9(69 \%)$ & $0.01^{\mathrm{b}}$ \\
\hline Centimetres from anal verge (SD) & $10.0(4.4)$ & $6.5(4.4)$ & $0.01^{\mathrm{a}}$ \\
\hline Total preoperative risk factors (SD) & $2.6(1.5)$ & $3.3(1.1)$ & $0.10^{\mathrm{a}}$ \\
\hline Intra-operative vasoactive drugs & $19(30 \%)$ & $7(54 \%)$ & $0.06^{\mathrm{b}}$ \\
\hline Intra-operative oliguria & $13(21 \%)$ & $5(38 \%)$ & $0.08^{\mathrm{b}}$ \\
\hline Splenic flexure mobilized & $19(30 \%)$ & $7(54 \%)$ & $0.11^{\mathrm{a}}$ \\
\hline Defunctioning stoma & $9(14 \%)$ & $5(38 \%)$ & $0.06^{\mathrm{b}}$ \\
\hline Laparoscopic procedure & $14(22 \%)$ & 0 & $0.06^{\mathrm{b}}$ \\
\hline
\end{tabular}

Numbers are depicted in mean (SD). Percentages between parentheses indicate the percentage of all patients with or without leakage

${ }^{\text {a }}$ Student's $t$ test

${ }^{\mathrm{b}}$ Chi-square test

A multiple regression analysis was performed on preoperative and intra-operative factors associated with anastomotic leakage (Tables 4 and 5). Duration of operation was associated with anastomotic leakage of anastomoses above $15 \mathrm{~cm}$ of the anal verge, in the ascending, transverse and descending colons. For anastomoses within $15 \mathrm{~cm}$ of the anal verge, mobilisation of splenic flexure, more distal anastomoses, oliguria during and after the operation and cardiac comorbidity were independent predictors of anastomotic leakage. When anastomotic height was not entered into regression analysis, the use of intra-operative vasoactive drugs and preoperative irradiation were significantly

Table 5 Independent predictors of anastomotic leakage analysed by multiple regression analysis (stepwise analysis)

\begin{tabular}{lrll}
\hline & \multicolumn{1}{c}{$B$} & $95 \%$ CI & $p$ \\
\hline $\begin{array}{l}\text { Anastomoses }>15 \mathrm{~cm}(n=115) \\
\text { Duration of operation }\end{array}$ & 0.002 & $0.001-0.003$ & $<0.001$ \\
$\begin{array}{l}\text { Anastomoses } \leq 15 \mathrm{~cm}(n=76) \\
\text { Mobilization of splenic flexure }\end{array}$ & 0.26 & $0.09-0.42$ & 0.04 \\
Centimeters from anal verge & -0.31 & -0.49 to 0.13 & 0.001 \\
Oliguria & 0.19 & $0.01-0.38$ & 0.04 \\
Cardiac comorbidity & 0.19 & $0.01-0.37$ & 0.04 \\
\hline
\end{tabular}

associated with anastomotic leakage. The number of patient-related risk factors for patients without and with anastomotic leakage are depicted in Fig. 4. Patients with an anastomotic leakage showed a higher rate in the number of risk factors compared to non-anastomotic leakage (5.8 SD 2.5 vs $4.6 \mathrm{SD} 2.2, p=0.02$ ).

The accuracy of the surgeons' estimation of risk for anastomotic leakage is shown in a receiver-operator characteristics curve for anastomoses $>/ \leq 15 \mathrm{~cm}$ from the anal verge (Fig. 5). When considering the median $(7.1 \%>15 \mathrm{~cm}, 9.5 \% \leq 15 \mathrm{~cm}$ from the anal verge) as the cut-off point for elevated risk in the surgeons' prediction of anastomotic leakage, sensitivity was $38 \%$ for high anastomosis and $62 \%$ for low anastomosis, and specificity was $46 \%$ and $52 \%$, respectively, for an accurate risk assessment of anastomotic leakage. When comparing assistants and surgeons, sensitivity was 44\% (median VAS $\leq 8.5 \%$ ) and $41 \%$ (median $\mathrm{VAS} \leq 7.8 \%$ ), specificity $59 \%$ and $48 \%(p=0.20)$, respectively.

\section{Discussion}

In this study, we evaluated the accuracy of the surgeons' judgement in prediction of anastomotic leakage in colorec- 
Fig. 4 The number of risk factors present in patients with (light bars, mean 5.8 SD 2.2) and without (dark bars, mean 4.6 SD 2.2) anastomotic leakage are depicted $(p=0.02)$. Error bars represent standard deviation

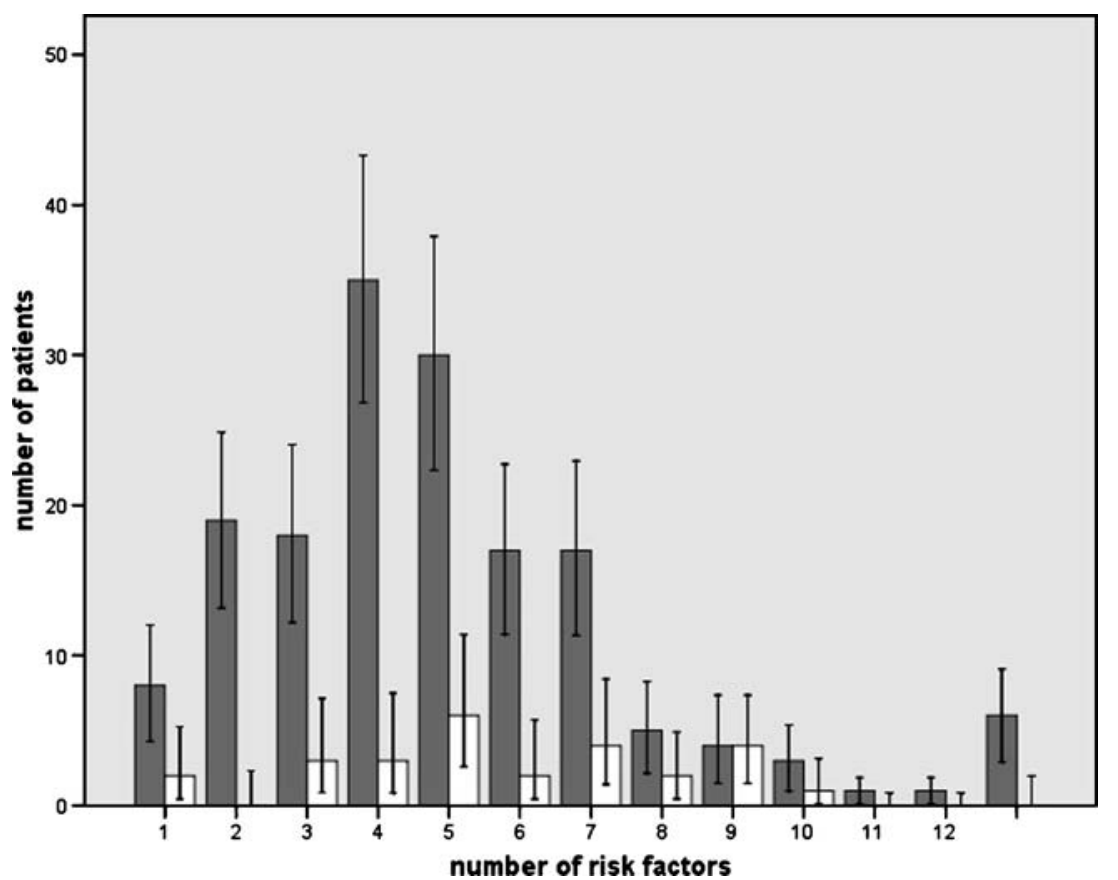

tal anastomoses. The clinical judgement of the operating surgeon in our study appeared to have low sensitivity and specificity for all anastomoses. When taking into account the relatively low exposure to anastomotic leakage of individual surgeons (i.e. a mean of $13.6 \%$ in this study), it might not be surprising that anastomotic leakage is difficult to predict (i.e. having a low a priori risk), thus resulting in a low post-test odds $(11 \%)$ of correctly predicting anastomotic leakage. The results of our study clearly identify the lack of a reliable intra-operative predictive test for anastomotic leakage by the operating surgeon, whereas several studies identify a large number of risk factors for anastomotic leakage. In general, the global, clinical judgement seems to localize a subset of patients at risk for developing complications in general, whereas many patients with no risk factors at all may develop anastomotic leakage [12].

The risk factors for anastomotic leakage identified by univariate and multivariate analysis in our study corroborate with those found in other studies [1-8]. Similar to these studies, the number of risk factors present in an individual patient appeared to be an important predictor of anastomotic leakage in all anastomoses. This finding was previously described in two large studies [1, 15]. Mäkelä et al. described leakage rates of $76 \%$ in patients with three risk factors, $87 \%$ with four risk factors and $100 \%$ in patients with five risk factors [15]. In Alves' study, leakage rates increased from $38 \%$, when two risk factors were present, to
$50 \%$ in the presence of three risk factors [1]. We found a similar increase in risk of anastomotic leakage with increasing risk factors, although the risk did not increase linearly. Furthermore, data on the weight of individual risk factors and the total amount of risk factors considered relevant are not readily available in the other two studies addressing this issue.

When considering individual risk factors for anastomotic leakage, it should be emphasized that construction of a defunctioning stoma and mobilisation of the splenic flexure might be considered a risk factor, as well as a measure to prevent anastomotic leakage. When conducting separate analysis for leakage rate and risk estimation, we found significantly higher leakage rates after mobilisation of the splenic flexure and construction of a stoma, showing that anastomotic leakage is not prevented by these measures. Moreover, the surgeons did not report a higher estimated risk for leakage in these patients on the basis of the visual analogue scale. This might point towards an underestimation of the leakage rate by the surgeon after mobilisation of the splenic flexure and construction of a defunctioning stoma. Another explanation might be that these procedures are mainly carried out in anastomoses within $15 \mathrm{~cm}$ of the anal verge and therefore present an epiphenomenon instead of a true causative relationship.

Markus et al. evaluated the clinical judgement of the operating surgeon (denominated as 'gut feeling') as a predictor for general post-operative mortality and morbidity 

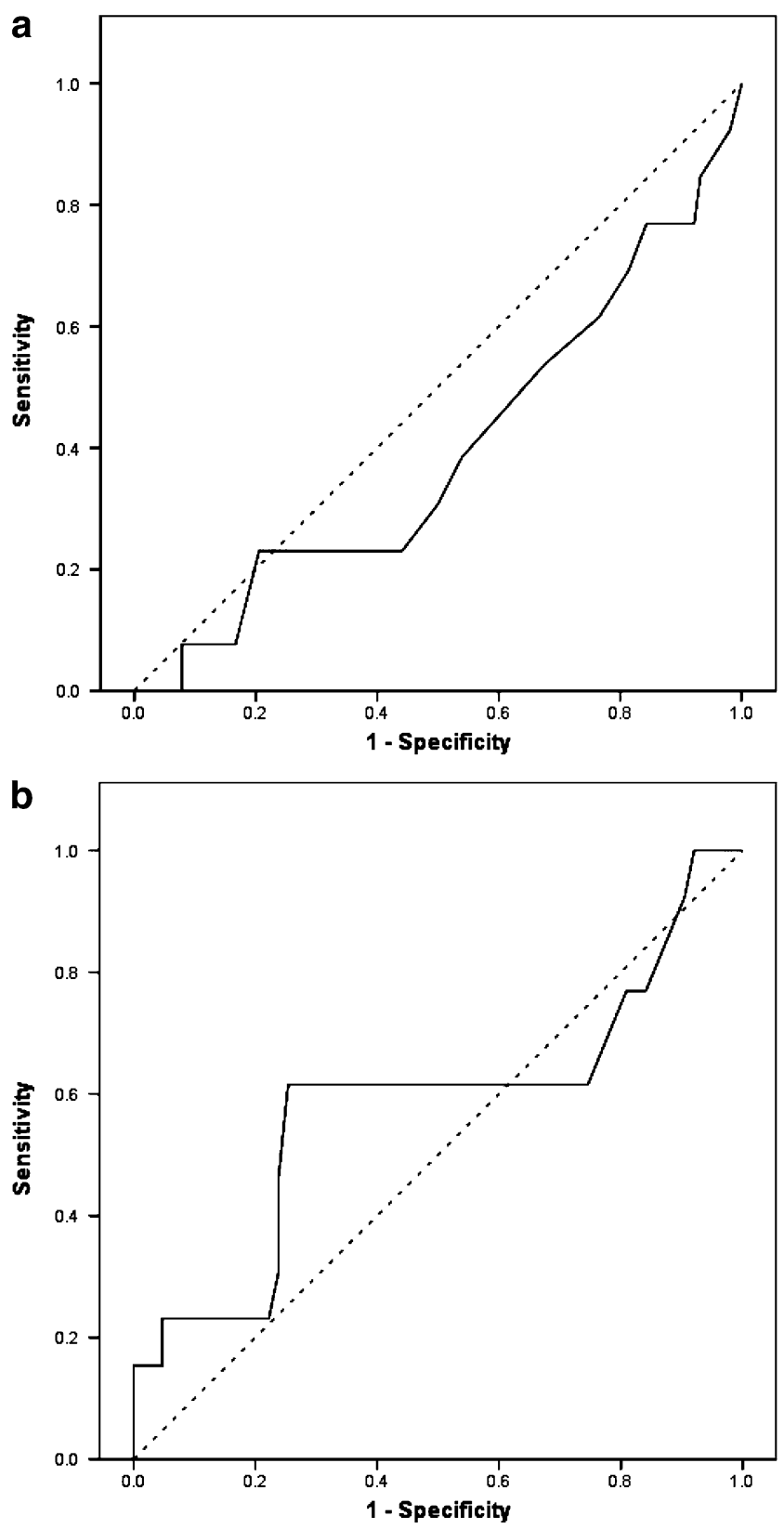

Fig. 5 Receiver-operator characteristics curve of probability estimation of anastomotic leakage by the surgeon for anastomoses $>15 \mathrm{~cm}$ (a) and $\leq 15 \mathrm{~cm}$ (b) from the anal verge

in large surgical interventions, comparing clinical judgement and prediction by POSSUM scores to actual outcome [14]. In this study, the surgeons' general clinical judgement was more accurate than POSSUM scores in upper gastrointestinal and hepatobiliary procedures but not in colorectal procedures. Pettigrew et al. compared the predictive value of the judgement of the operating surgeon for development of post-operative complications based on a global assessment of the patient, with the assessment of an independent physician performing a complete physical examination and with several anthropometric indices [13]. In a population of 218 patients undergoing major gastrointestinal surgery, they reported that global risk assessment by the operating surgeon is less accurate (sensitivity $32 \%$, specificity $83 \%$ ) than careful assessment of in particular cardiorespiratory disease and nutritional status of the patient. In a third study using a similar design [12] but evaluating the surgeons' judgement before and after surgery as well, they concluded the surgeons' post-operative judgement to be superior to the surgeons' preoperative judgement or assessment of either preoperative patient factors or systematic clinical factors. Based on this striking finding, they concluded that surgical performance during the operation is an important predictor of post-operative complications. Considering this latter study [12], it might be assumed that the surgeon is able to reliably predict the risk of anastomotic leakage, but as mentioned by Markus et al.[14], they found the surgeons' risk estimation after completion of the operation not to be a good predictor of post-operative complications, particularly in colorectal surgery. This finding is emphasized by our study. One should be aware that none of the aforementioned studies addresses anastomotic leakage as primary end point in particular. Furthermore, it needs to be addressed that VAS has not been used before to estimate leakage rates and should therefore be considered a rough measure. However, we used the VAS in the design of this study as a more reliable estimation than simply asking the surgeon to estimate the risk of leakage as a percentage [15].

In conclusion, this prospective study has shown that global clinical risk assessment of anastomotic leakage by the operating surgeon has low predictive value for anastomotic leakage and underestimates the risk of anastomotic leakage. This clearly supports the need for the development of a more reliable predictive test. Such a test should be carried out intra-operatively, real-time and easy to acquire, to interpret and to act on. Moreover, the test should have a high sensitivity for anastomotic leakage because of the detrimental effect of missing a leak. When taking the possible pathophysiological mechanism of anastomotic leakage into account [16, 17], probably a diagnostic test which measures microperfusion before and after creation of an anastomosis will be most suitable. At our centre, we are developing optical imaging techniques such as visible light spectroscopy $[18,19]$ and multispectral fluorescence imaging techniques [20] to evaluate realtime microperfusion in gastrointestinal surgery. Future (pre)clinical studies will elucidate the value of these techniques. 
Open Access This article is distributed under the terms of the Creative Commons Attribution Noncommercial License which permits any noncommercial use, distribution, and reproduction in any medium, provided the original author(s) and source are credited.

\section{References}

1. Alves A, Panis Y, Trancart D et al (2002) Factors associated with clinically significant anastomotic leakage after large bowel resection: multivariate analysis of 707 patients. World J Surg 26:499-502

2. Ptok H, Marusch F, Meyer F et al (2007) Impact of anastomotic leakage on oncological outcome after rectal cancer resection. Br J Surg 94:1548-1554

3. Eriksen MT, Wibe A, Norstein J et al (2005) Norwegian Rectal Cancer Group. Anastomotic leakage following routine mesorectal excision for rectal cancer in a national cohort of patients. Colorectal Dis 7:51-57

4. Karl RC, Schreiber R, Boulware D et al (2000) Factors affecting morbidity, mortality and survival in patients undergoing Ivor Lewis esophagogastrectomy. Ann Surg 5:635-643

5. Viklund P, Lindblad M, Lu M et al (2006) Risk factors for complications after esophageal cancer resection: a prospective population-based study. Ann Surg 243:204-211

6. Vignali A, Fazio VW, Lavery IC et al (1997) Factors associated with the occurrence of leaks in stapled rectal anastomoses: a review of 1014 patients. J Am Coll Surg 185:113-121

7. Matthiessen P, Hallböök O, Andersson M et al (2004) Risk factors for anastomotic leakage after anterior resection of the rectum. Colorectal Dis 6:462-469

8. Katory M, Tang CL, Koh WL, Fook-Chong SMC, Loi TT, Ooi BS, Ho KS, Eu KW (2008) A 6-year review of surgical morbidity and oncological outcome after high anterior resection for colorectal malignancy with and without splenic mobilisation. Colorectal Dis 10:165-169
9. Matthiessen P, Hallböök O, Rutegård J, Simert G, Sjödahl R (2007) Defunctioning stoma reduces symptomatic anastomotic leakage after low anterior resection of the rectum for cancer: a randomized multicenter trial. Ann Surg 246:207-214

10. Gastinger I, Marusch F, Steinert R, Wolff S, Koeckerling F, Lippert H, Working Group 'Colon/Rectum Carcinoma' (2005) Protective defunctioning stoma in low anterior resection for rectal carcinoma. Br J Surg 92:1137-1142

11. Peeters KC, Tollenaar RA, Marijnen CA, Klein Kranenbarg E, Steup WH, Wiggers T, Rutten HJ, van de Velde CJ, Dutch Colorectal Cancer Group (2005) Risk factors for anastomotic failure after total mesorectal excision of rectal cancer. Br J Surg 92:211-216

12. Pettigrew RA, Burns HJG, Carter DC (1987) Evaluating surgical risk: the importance of technical factors in determining outcome. Br J Surg 74:791-794

13. Pettigrew RA, Hill GL (1986) Indicators of surgical risk and clinical judgement. Br J Surg 73:47-51

14. Markus PM, Martell J, Leister I, Horstmann O, Brinker J, Becker H (2005) Predicting postoperative morbidity by clinical assessment. Br J Surg 92:101-106

15. Patrician PA (2004) Single-item graphic representational scales. Nurs Res 53:347-352

16. Makela JT, Kiviniemi H, Laitinen S (2003) Risk factors for anastomotic leakage after left sided colorectal resection with rectal anastomosis. Dis Colon Rectum 46:653-660

17. Stokes KY, Granger DN (2005) The microcirculation: a motor for the systemic inflammatory response and large vessel disease induced by hypercholesterolaemia? J Physiol 562:647-653

18. Benaron DA, Parachikov IH, Friedland S et al (2004) Continuous, noninvasive and localised microvascular tissue oximetry using visible light spectroscopy. Anesthesiology 100:1469-1475

19. Friedland SR, Benaron D (2004) Reflectance spectrophotometry for the assessment of mucosal perfusion in the gastrointestinal tract. Gastrointest Endosc Clin N Am 14:539-555

20. Weissleder R, Pittet MJ (2008) Imaging in the era of molecular oncology. Nature 452:580-589 